\title{
Lipidomics in drug development
}

\section{Jonas Dehairs', Rita Derua², Natalia Rueda-Rincon', Johannes V. Swinnen ${ }^{1, *}$}

'KU Leuven - University of Leuven, Department of Oncology, Laboratory of Lipid Metabolism and Cancer, B-3000 Leuven, Belgium

${ }^{2} \mathrm{KU}$ Leuven - University of Leuven, Department of Cellular and Molecular Medicine, Laboratory of Protein Phosphorylation and

Proteomics, B-3000 Leuven, Belgium

Numerous human pathologies, including common conditions such as obesity, diabetes, cardiovascular disease, cancer, inflammatory disease and neurodegeneration, involve changes in lipid metabolism. Likewise, a growing number of drugs are being developed that directly or indirectly affect lipid metabolic pathways. Instead of classical and cumbrous radiochemical analyses, lipid profiling by mass spectrometry (MS)-based lipidomics holds great potential as companion diagnostic in several steps along the drug development process. In this review we describe some typical lipidomics set-ups and illustrate how these technologies can be implemented in target discovery, compound screening, in vitro and in vivo preclinical testing, toxicity testing of drugs, and prediction and monitoring of response.

\section{Introduction}

With more than 180,000 different molecular species, lipids form a vast and extraordinary class of biomolecules. Each of these lipids has a unique chemical structure and biophysical properties. Functioning as structural components of membranes, as medium for energy storage, as anchor for proteins, or as intra- and inter-cellular signaling molecules, lipids play diverse roles and are involved in numerous biological processes [1,2]. The total lipid composition of an organism or specimen thereof is referred to as the lipidome. The lipidome is affected by the diet and is further controlled by a large and diverse family of lipid metabolic enzymes, which are tightly

*Corresponding author: J.V. Swinnen (johan.swinnen@med.kuleuven.be)

\section{Section editor:}

Pascal de Tullio - University of Liège, Liège, Belgium.

regulated by both central and cell-autonomous control mechanisms. Not surprisingly, many human diseases, including common conditions such as obesity, diabetes, cardiovascular disease, inflammatory disease, cancer and neurodegeneration involve changes in lipids and in lipid metabolic pathways [3-6]. Likewise, an increasing number of drugs have been or are being developed that directly or indirectly target lipid metabolism. Established or emerging targets include enzymes involved in the synthesis and metabolism of cholesterol, fatty acids, phospholipids, sphingolipids and triglycerides (Fig. 1). Also their regulators, including key signaling proteins and transcription factors have been identified as potential targets in the pathologies mentioned above. Until recently, discovery and validation of drugs targeting these pathways was largely based on the use of radiolabeled substrates. The methodologies involved are often cumbersome, do not allow multiplexing, often lack specificity, and entail safety and environmental risks due to the use of radiolabels. Recent advances in technologies such as spectroscopy, chromatography and most importantly in mass spectrometry, have overcome these shortcomings and have established lipidomics as a maturing 'omic' within the 'metabolomics' field and alongside other systems biology approaches including genomics, transcriptomics, and proteomics. Introduction of these technologies into the various stages of the drug development process provides unparalleled possibilities all the way from compound screening to clinical diagnostics (Fig. 2). 


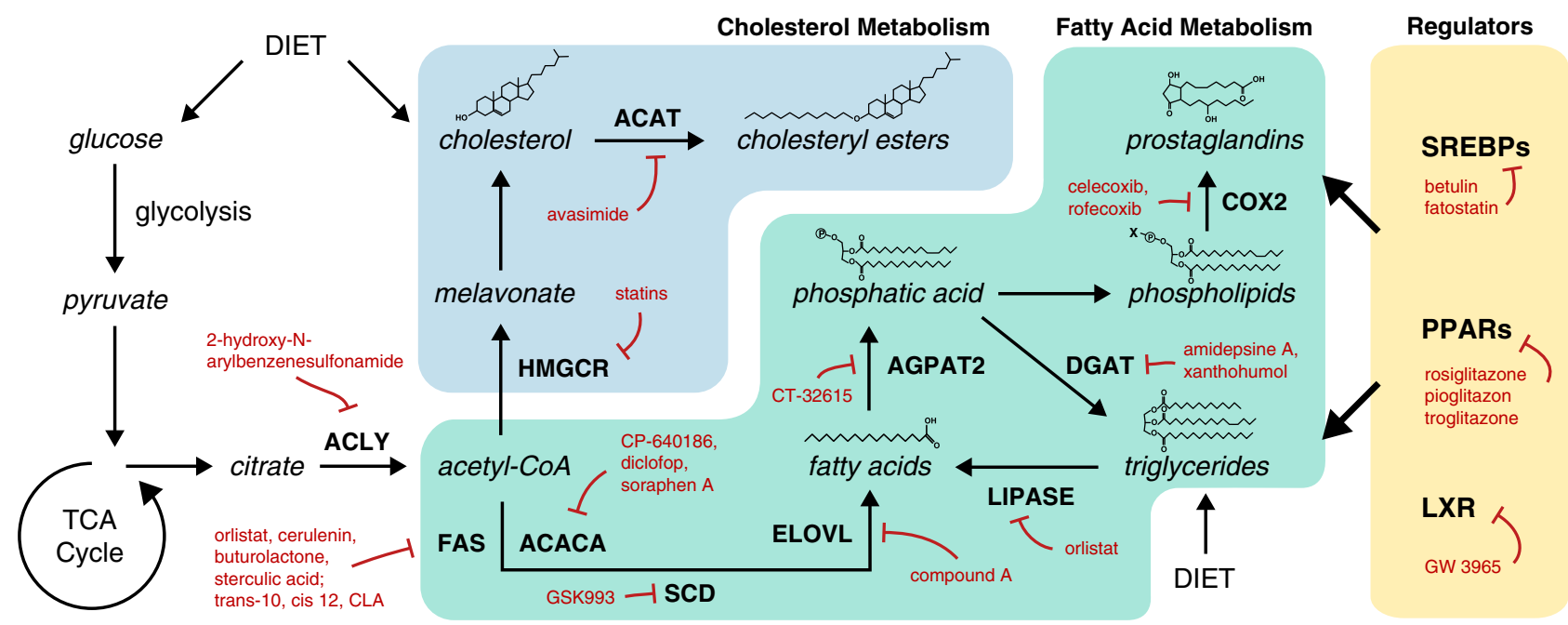

Drug Discovery Today: Technologies

Figure I. Lipid metabolic enzymes as targets for drug development. A selection is shown of enzymes and regulators of lipid metabolic pathways that are currently being used or explored as drug targets, along with some representative drugs or modulating compounds (indicated in red). ACAT, acetylcoenzyme A acetyltransferase; ACLY, ATP citrate lyase; ACACA, acetyl-CoA carboxylase; AGPAT2, I-acylglycerol-3-phosphate O-acyltransferase 2; COX2, prostaglandin-endoperoxide synthase 2; DGAT, diglyceride acyltransferase; ELOVL, elongation of very long chain; FAS, fatty acid synthase; HMGCR, 3-hydroxy-3-methylglutaryl-CoA reductase; LXR, liver X receptor; PPARs, peroxisome proliferator-activated receptors; SCD, stearoyl-CoA desaturase; SREBPs, sterol regulatory element-binding proteins.

\section{Analytical methods to study lipids}

Due to the large structural complexity and heterogeneity of lipids compared to DNA, RNA and even proteins, lipidomics has been lagging behind other omics approaches in terms of throughput and comprehensiveness. Early lipid separation and analysis methods are largely based on chromatographic separation techniques including thin layer chromatography (TLC), high performance liquid chromatography (HPLC) and gas chromatography (GC). The latter methods excel by their easy set up but suffer from a limited resolving capacity hampering the analysis of individual lipid species. GC is more performing in this context but has the disadvantage that it is not amenable to complex mixtures, which often have to be hydrolyzed and derivatized. Other options include nuclear magnetic resonance (NMR) spectroscopy and Raman

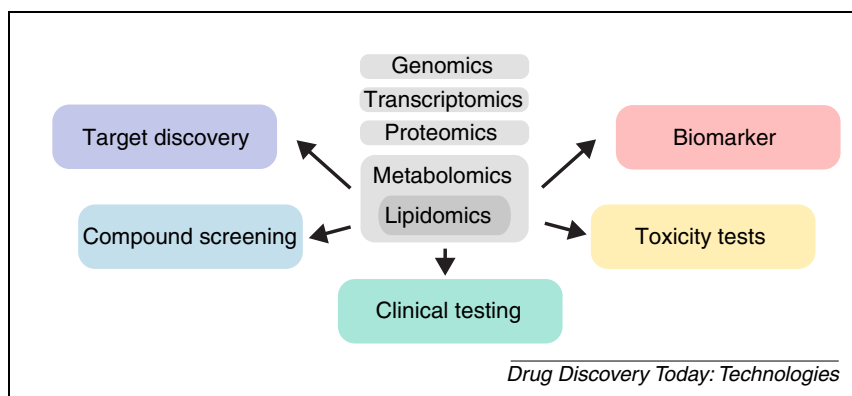

Figure 2. Situation of lipidomics in the 'omics' landscape and its implementation as companion diagnostic in several steps along the drug development process. spectroscopy. Both methods have their merits in the lipidomics field, but fall short in terms of sensitivity and resolution. Progress in mass spectrometry (MS), especially in high-resolution instruments, has enabled to increase sensitivity, specificity and throughput of lipidomic screens from complex biological mixtures and to execute structural characterization of individual molecular species in tandem MS experiments. Therefore, MS has become the prime method for comprehensive lipid analysis, often in combination with the separation techniques mentioned above.

\section{Mass spectrometry-based lipidomics}

Mass spectrometry is an analytical technique that allows the identification, structural characterization and quantification of biomolecules based on the mass-to-charge ratio $(\mathrm{m} /$ $z)$ of gas-phase ions. Typically, lipid extracts are prepared from biological samples using organic solvents, traditionally using a mixture of methanol and chloroform [7] or, more recently, using methyl-tert-butyl ether (MTBE) [8] (Fig. 3). Crude lipid extracts can then directly be subjected to mass spectrometry (defined as shotgun-lipidomics) [9] or first be separated by liquid or gas chromatography. Prior separation of lipids reduces ion suppression and minimizes the overlap of lipid species with the same nominal or exact mass (isobaric and isomeric lipid species). On the other hand, it is more time-consuming and often less quantitative suffering from variable solvent/analyte ratios. As mentioned above, gas chromatography requires hydrolyzation and derivatization of molecules. 


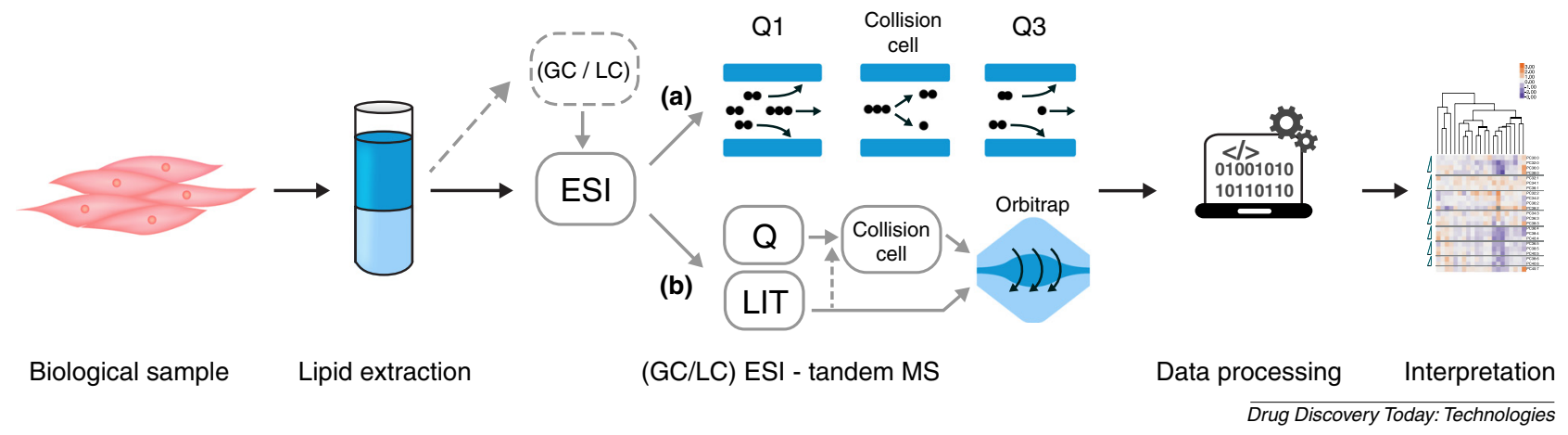

Figure 3. Typical set-ups for tandem MS-based lipidomics. Biological samples (tissues, body fluids, etc.) are subjected to lipid extraction. Lipids are then separated by LC or GC, or directly infused into ESI-tandem MS instruments. (a) A low-resolution setup consisting of two quadrupole mass analyzers (QI and Q3) separated by a collision cell (Q2). Tandem MS scans are used for identification and quantification of molecular species. (b) A high-resolution setup consisting of an orbitrap mass analyzer interfaced to a second mass analyzer ( $Q$ or LIT) and a collision cell (if appropriate). Full MS scans are used for identification and quantification of molecular species, with tandem MS scans targeting only a small number of selected precursors. Data are visualized for example as heatmaps or subjected to further statistical analysis.

Before entering the mass spectrometer, lipid molecules (either as crude extracts or after separation) are subjected to soft ionization techniques (avoiding extensive in-source fragmentation) like electrospray ionization (ESI) or matrixassisted laser desorption ionization (MALDI). In ESI, lipids in solution acquire positive or negative charges by charged droplet formation followed by desolvation, whereas in MALDI, lipids are co-crystallized with a matrix, which absorbs and transfers energy from a laser beam to the lipid molecules, resulting in their ionization. In the context of lipidomics, the latter technique is mainly used for MS-based lipid imaging in tissue sections.

Detection of ionized lipids is performed by single MS or tandem MS, making use of one or two mass analyzers, respectively. Tandem MS instruments consist of two mass analyzers separated by a collision cell, and are capable of operating in different MS/MS scanning modes (product ion scan, precursor ion scan, neutral loss scan, MRM), producing structurespecific fragment ions (Fig. 3). The tandem MS scan displaying the highest sensitivity, specificity and efficiency in terms of duty cycle is the multiple reaction monitoring (MRM) scan, producing only a signal if predetermined precursor $(\mathrm{m} / \mathrm{z}) /$ fragment $(\mathrm{m} / \mathrm{z})$ pairs are detected. Most shotgun lipidomics experiments on low resolution mass spectrometers make use of tandem MS scans for identification and quantification of molecular species (bottom-up shotgun lipidomics) [10]. Although targeted, MRM is often the tandem MS method of choice for lipid quantification. On the other hand, Fourier transform-based mass spectrometers (FTMS), characterized by high resolution and mass accuracy, are able in single MS mode to detect and quantify global changes in lipid composition directly from crude lipid extracts, solely based on the lipid intact mass $(\mathrm{m} / \mathrm{z})$ (top-down shotgun lipidomics), leaving tandem MS for a small number of selected precursors [11]. The advent of easy-to-operate and robust hybrid Orbitrap technology, has brought FTMS into the lipidomics laboratory. Other techniques that can add an orthogonal separation dimension to a standard shotgun lipidomics workflow are ion mobility spectrometry [12] and differential mobility spectrometry (DMS) [13], enabling the separation of isobaric and isomeric lipids in front of the mass spectrometer.

During processing, the data are subjected to spectral filtering, chromatographic alignment (in case of LC), corrections for ${ }^{13} \mathrm{C}$ isotope effects, quantitation through ratio comparison of ion peaks to internal standard peaks, visualization and export of data (Fig. 3). Several software packages have been developed to aid in processing of data [14-16]. The paucity of individual calibration standards to determine the response coefficient of each molecular species limits the absolute quantification potential of the method. Moreover the wide variety of approaches used by different researchers is hampering easy exchange of data and is calling for a further standardization of the technology. Further biostatistical analysis usually involves multivariate analysis, such as principal component analysis (PCA), which is useful for the identification of correlations of lipid metabolites with pathological phenotypes, for instance for the development of lipid-based biomarkers.

An interesting application involves the use of stable isotope-labeled precursor metabolites such as glucose, whose incorporation into lipid can be followed by MS or NMR. This approach allows metabolic pathway elucidation and can be applied to in vivo models and patients $[17,18]$.

\section{Lipidomics in target discovery}

Since its development, MS-based lipidomics has been applied for the analysis of lipids in numerous disease conditions. Analysis can be carried out on tissues or body fluids of diseased and healthy human subjects as well as on disease models involving animals or cultured cells. Both LC-MS and 

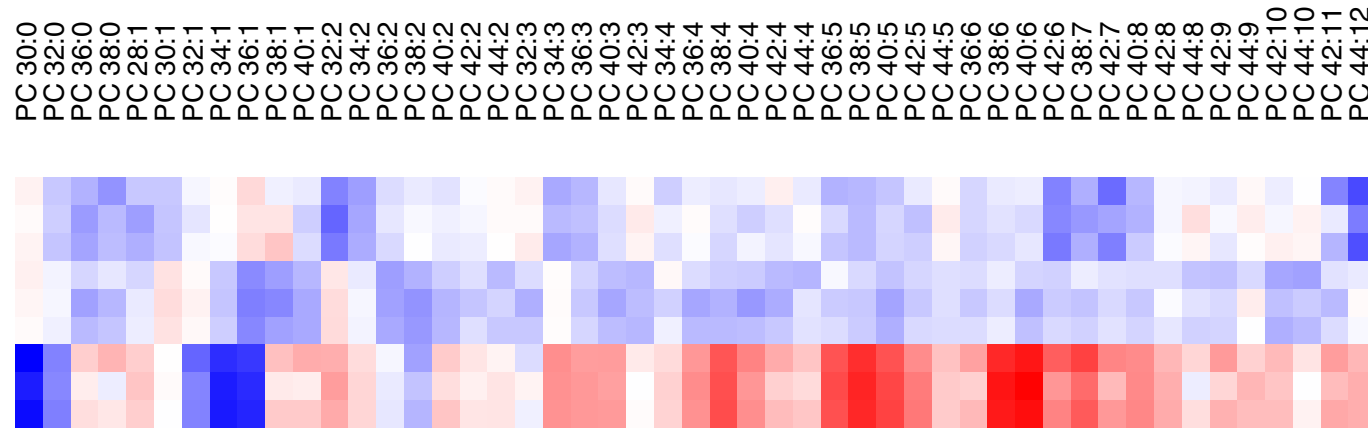

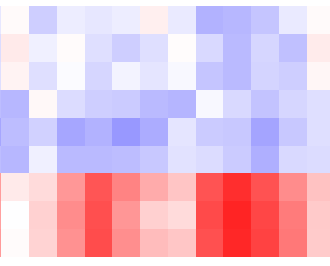

$\log _{2}$ (inhibitor / control)

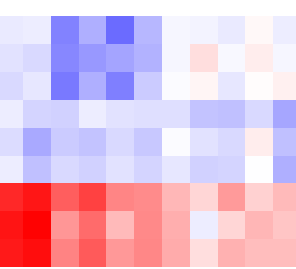

$-2$

3.5

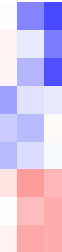

SCD

SCD1

ELOVL6

ELOVL6

ELOVL6

ACACA

ACACA

ACACA

Drug Discovery Today: Technologies

Figure 4. Identification of drugs and drug targets based on the unique changes in lipid profiles each compound produces. Cancer cells (HCTII6) treated with different compounds, for example an SCDI inhibitor, an ELOVL6 inhibitor or an ACACA inhibitor show unique and characteristic changes in lipid profiles.

shotgun lipidomics have been very successful in this context and have led to the identification of hitherto unknown changes in lipid profiles. Besides new insights in classical lipid-related diseases such as cardiovascular disease and obesity, lipidomics analysis has revealed unsuspected changes in the lipidome in many other disease conditions. Striking examples include the discovery of recurrent changes in the phospholipidome of cancer tissues [19-23] and in sphingolipids in Alzheimer's disease [24,25]. Combined with transcriptome analysis and other functional genetics tools, these findings have led to new insights in disease mechanisms and to the discovery of potential new targets for drug development [23,26-29].

\section{Lipidomics in the identification of candidate drugs}

Identification of novel drug candidates often involves the screening of thousands of chemical compounds. In the case of lipid metabolizing enzymes, assays involving radiolabeled substrates are typically used. Also here, MS-based lipidomics holds great potential as it enables simultaneous and highly selective analysis of natural intermediates and end products, as well as side products due to off-target effects, thus overcoming many of the limitations inherently associated with the use of radiolabeled substrates. However, standard lipidomic analyses have a limited throughput due to pre-MS sample preparation involving liquid extractions, chromatographic separations, sample injection and post-MS -data processing. Thus far this has restricted the use of lipidomics approaches largely to low or medium throughput projects mainly in hitto-lead (H2L) and lead optimization (LO) phases of the drug discovery process. Recent advances in MS instrumentation and particularly in the automation of pre- and post-MS handling of samples and data can dramatically increase the throughput of the analysis, enabling the use of MS-based lipidomics for larger compound screening projects. Particularly, the capacity of lipid extraction can be substantially increased by the use of robotic liquid handling stations and can be further improved by solid phase extraction (SPE). This involves the use of a solid matrix for which the analytes to be isolated (lipids in this case) have a high affinity, allowing their selective separation and elution. SPE can be adapted to isolate a broad range of lipids either in batch or as separate fractions after differential elution [30]. Sample injection can also be automated by using robotic sample injection and ionization devices. One example of such systems is the TriVersa NanoMate (Advion), which is routinely used in the author's facility. It employs microfluidics chips containing an array of up to 384 nanoelectrospray nozzles per chip, allowing reproducible sample injection and spraying, while avoiding cross-contamination between samples. Shotgun lipidomics is the approach of choice for high throughput applications (vide supra). Recently, high throughput mass spectrometry devices with or without parallel processing capacities have become available allowing unattended analysis of thousands of samples [31]. Also data analysis can be automated and software can be equipped with built-in profile or pattern recognition. As each lipid metabolic enzyme possesses specific activities, unique changes in profiles are generated upon chemical inhibition or elimination of a specific enzyme (Fig. 4). By comparing lipid profiles generated in compound screenings with available profiles of selective inhibition or knockdown of individual enzymes or their regulators, active compounds can be identified and their targets be predicted.

\section{Lipidomics in preclinical and clinical testing}

Any drug development program must proceed through several steps in order to deliver a final product that is efficacious and safe. Essential is the monitoring of biological effects needed for decision making in early drug development. To this end so-called pharmacodynamics (PD) markers are needed that are used in pre-clinical and clinical studies to establish doses and dosing regimens for subsequent studies. In the case of lipid metabolic enzymes, enzyme activity is often 
monitored by measuring the incorporation of radioactive tracers into intermediate or end products. As an example, the incorporation of ${ }^{14} \mathrm{C}$ acetate into lipids in cell cultures can be used to monitor the efficacy of fatty acid synthesis inhibitors. In vivo and particularly in phase I clinical studies the use of radioactive tracers is often less obvious, not only due to the use of radiolabels, but particularly because of the more complex metabolism of many tracers at the level of an entire organism. This emphasizes the need for alternative assays. Also at this stage, MS-based lipid profiling may offer an attractive solution. Depending on the target, small tissue biopsies and/or body fluids can be taken and analyzed for changes in the lipidome. Not only can changes in the lipidome be assessed from total lipid extracts, they can also be verified in multidimensional tissue sections by MALDI-MSbased lipid imaging, providing information about the spatial localization of the alterations [32]. Dynamic changes and alteration of fluxes through lipid metabolic pathways can be followed by simultaneous treatment with stable isotopelabeled compounds. As many lipid species can be monitored simultaneously, MS-based lipidomics has the added advantage of providing an indication on off-target effects and toxicity (vide infra), in addition to information on the efficacy of the compound.

\section{Lipidomics for companion biomarker analysis}

In an era of strong emphasis on personalized medicine, biomarkers that have the potential to select patients that are most amenable for a specific drug, that can monitor the efficacy of the drug and that can predict toxicity are of utmost importance. MS-based lipidomics has great potential in all these areas. Numerous lipidomics studies have identified lipid species that aid in the early detection and/or differential diagnosis of diseases $[5,33,34]$. Toxicity, particularly hepatotoxicity evoked by drugs has been successfully detected using lipidomics approaches [35,36]. Similarly, lipid analysis has been used to monitor the response to several drugs $[37,38]$. Instead of using tissue biopsies for analysis, the high sensitivity of most MS-based approaches, enable the analysis of body fluids, which offer the advantage of minimal invasiveness and allow repeated sampling. Selective enrichment or isolation of circulating cells or other fractions from body fluids often provide solutions when sensitivity is an issue. Of particular interest in this context is the isolation of exosomes from body fluids. Exosomes are small vesicles that are released from cells and that can be found at high levels in body fluids $[39,40]$. As many disease markers, including aberrant lipid profiles, that are present in cells and tissues can also be found in exosome fractions [21,41,42], lipidomics analysis of exosomes is a booming area of research that holds great potential in the context of companion biomarker development.

\section{Conclusions and future perspectives}

In view of the increasing world-wide incidence of classical lipid-related diseases, including obesity, diabetes, and metabolic syndrome, together with the growing awareness that other common diseases such as cancer and neurodegenerative disorders are accompanied by alterations in lipid metabolism, an increasing number of drugs development programs are focusing on enzymes and regulators involved in lipid metabolism. From this brief overview and the increasing amount of literature, it is clear that MS-based lipidomics is a rapidly emerging technology that holds great potential as companion diagnostic tool along all stages of the drug development process (Fig. 2). Ongoing developments aimed at increasing the throughput of this technology through further automation, along with further standardization will undoubtedly place MS-based lipidomics at the heart of many drug development programs.

\section{Conflict of interest}

The authors declare no conflict of interest.

\section{Acknowledgements}

Lipidomics developments in the author's facilities were in part supported by grants from the Foundation Fournier Majoie (FFM), the Research Foundation Flanders (FWO) and Concerted Research Action (GOA) Programs by the KU Leuven. J.D. is recipient of a research fellowship from the Flemish Agency for Innovation by Science and Technology (IWT).

\section{References}

1. Thiam AR, Farese RV, Walther TC. The biophysics and cell biology of lipid droplets. Nat Rev Mol Cell Biol 2013;14:775-86.

2. Van Meer G, Voelker DR, Feigenson GW. Membrane lipids: where they are and how they behave. Nat Rev Mol Cell Biol 2008;9:112-24.

3. Santos CR, Schulze A. Lipid metabolism in cancer. FEBS J 2012;279:2610_ 23

4. Wymann MP, Schneiter R. Lipid signalling in disease. Nat Rev Mol Cell Biol 2008;9:162-76.

5. Mapstone M, Cheema AK, Fiandaca MS, Zhong X, Mhyre TR, MacArthur LH, et al. Plasma phospholipids identify antecedent memory impairment in older adults. Nat Med 2014;20:415-8.

6. Harrison C. Obesity and diabetes: targeting lipid transport in diabetes. Nat Rev Drug Discov 2012;11:908.

7. Bligh EG, Dyer WJ. A rapid method of total lipid extraction and purification. Can J Biochem Physiol 1959;37:911-7.

8. Matyash V, Liebisch G, Kurzchalia TV, Shevchenko A, Schwudke D. Lipid extraction by methyl-tert-butyl ether for high-throughput lipidomics. J Lipid Res 2008;49:1137-46.

9. Han X, Gross RW. Shotgun lipidomics: electrospray ionization mass spectrometric analysis and quantitation of cellular lipidomes directly from crude extracts of biological samples. Mass Spectrom Rev 2005;24:367-412.

10. Han X, Yang K, Gross RW. Multi-dimensional mass spectrometry-based shotgun lipidomics and novel strategies for lipidomic analyses. Mass Spectrom Rev 2012;31:134-78.

11. Schwudke D, Schuhmann K, Herzog R, Bornstein SR, Shevchenko A. Shotgun lipidomics on high resolution mass spectrometers. Cold Spring Harb Perspect Biol 2011;3. 
12. Kliman M, May JC, McLean JA. Lipid analysis and lipidomics by structurally selective ion mobility-mass spectrometry. Biochim Biophys Acta 2011;1811:935-45.

13. Lintonen TPI, Baker PRS, Suoniemi M, Ubhi BK, Koistinen KM, Duchoslav E, et al. Differential mobility spectrometry-driven shotgun lipidomics. Anal Chem 2014. http://dx.doi.org/10.1021/ac5021744.

14. Herzog R, Schuhmann K, Schwudke D, Sampaio JL, Bornstein SR, Schroeder M, et al. LipidXplorer: a software for consensual cross-platform lipidomics. PLoS ONE 2012;7:e29851.

15. Husen P, Tarasov K, Katafiasz M, Sokol E, Vogt J, Baumgart J, et al. Analysis of lipid experiments (ALEX): a software framework for analysis of highresolution shotgun lipidomics data. PLoS ONE 2013;8:e79736.

16. Kind T, Liu K-H, Lee DY, DeFelice B, Meissen JK, Fiehn O. LipidBlast in silico tandem mass spectrometry database for lipid identification. Nat Methods 2013;10:755-8.

17. Higashi RM, Fan TW-M, Lorkiewicz PK, Moseley HNB, Lane AN. Stable isotope-labeled tracers for metabolic pathway elucidation by GC-MS and FT-MS. Methods Mol Biol 2014;1198:147-67.

18. Lane AN, Fan TW-M, Bousamra M, Higashi RM, Yan J, Miller DM. Stable isotope-resolved metabolomics (SIRM) in cancer research with clinical application to nonsmall cell lung cancer. OMICS J Integr Biol 2011;15:173-82.

19. Marien E, Meister M, Muley T, Fieuws S, Bordel S, Derua R, et al. Non-small cell lung cancer (NSCLC) is characterized by dramatic changes in phospholipid profiles. Int J Cancer 2015. http://dx.doi.org/10.1002/ ijc.29517.

20. Dória ML, Ribeiro AS, Wang J, Cotrim CZ, Domingues P, Williams C, et al. Fatty acid and phospholipid biosynthetic pathways are regulated throughout mammary epithelial cell differentiation and correlate to breast cancer survival. FASEB J 2014. http://dx.doi.org/10.1096/fj.14-249672.

21. Llorente A, Skotland T, Sylvänne T, Kauhanen D, Róg T, Orłowski A, et al. Molecular lipidomics of exosomes released by PC-3 prostate cancer cells. Biochim Biophys Acta 2013;1831:1302-9.

22. Zhou X, Mao J, Ai J, Deng Y, Roth MR, Pound C, et al. Identification of plasma lipid biomarkers for prostate cancer by lipidomics and bioinformatics. PLoS ONE 2012;7:e48889.

23. Hilvo M, Denkert C, Lehtinen L, Müller B, Brockmöller S, Seppänen-Laakso $\mathrm{T}$, et al. Novel theranostic opportunities offered by characterization of altered membrane lipid metabolism in breast cancer progression. Cancer Res 2011;71:3236-45.

24. He X, Huang Y, Li B, Gong C-X, Schuchman EH. Deregulation of sphingolipid metabolism in Alzheimer's disease. Neurobiol Aging 2010;31:398-408.

25. Van Echten-Deckert G, Walter J. Sphingolipids: critical players in Alzheimer's disease. Prog Lipid Res 2012;51:378-93.

26. Schlaepfer IR, Rider LC, Ulkus Rodrigues L, Gijon MA, Pac CT, Romero L, et al. Lipid catabolism via CPT1 as a therapeutic target for prostate cancer. Mol Cancer Ther 2014. http://dx.doi.org/10.1158/1535-7163.MCT-14-0183.

27. Jiao J, Ishikawa T-O, Dumlao DS, Norris PC, Magyar CE, Mikulec C, et al. Targeted deletion and lipidomic analysis identify epithelial cell COX-2 as a major driver of chemically-induced skin cancer. Mol Cancer Res 2014. http://dx.doi.org/10.1158/1541-7786.MCR-14-0397-T.

28. Nomura DK, Long JZ, Niessen S, Hoover HS, Ng S-W, Cravatt BF. Monoacylglycerol lipase regulates a fatty acid network that promotes cancer pathogenesis. Cell 2010;140:49-61.

29. Liu Y, Chen Y, Momin A, Shaner R, Wang E, Bowen NJ, et al. Elevation of sulfatides in ovarian cancer: an integrated transcriptomic and lipidomic analysis including tissue-imaging mass spectrometry. Mol Cancer 2010;9:186.

30. Zou W, Zou W. Separation of lipids by solid phase extraction (SPE). Protoc Exch 2011. http://dx.doi.org/10.1038/protex.2011.246.

31. Jung HR, Sylvänne T, Koistinen KM, Tarasov K, Kauhanen D, Ekroos K. High throughput quantitative molecular lipidomics. Biochim Biophys Acta 2011;1811:925-34.

32. Manier ML, Spraggins JM, Reyzer ML, Norris JL, Caprioli RM. A derivatization and validation strategy for determining the spatial localization of endogenous amine metabolites in tissues using MALDI imaging mass spectrometry. J Mass Spectrom 2014;49:665-73.

33. Kulkarni H, Meikle PJ, Mamtani M, Weir JM, Barlow CK, Jowett JB, et al. Plasma lipidomic profile signature of hypertension in Mexican American families: specific role of diacylglycerols. Hypertension 2013;62:621-6.

34. Mayerle J, Kalthoff H, Reszka RC, Kamlage B, Peter E, Schniewind B, et al. Metabolic biomarkers for the differential diagnosis of pancreatic ductal adenocarcinoma vs. chronic pancreatitis. Cancer Metab 2014;2:P60.

35. Shi X, Yao D, Gosnell BA, Chen C. Lipidomic profiling reveals protective function of fatty acid oxidation in cocaine-induced hepatotoxicity. J Lipid Res 2012;53:2318-30.

36. Parman T, Bunin DI, Ng HH, McDunn JE, Wulff JE, Wang A, et al. Toxicogenomics and metabolomics of pentamethylchromanol (PMCol)induced hepatotoxicity. Toxicol Sci 2011;124:487-501.

37. Yetukuri L, Huopaniemi I, Koivuniemi A, Maranghi M, Hiukka A, Nygren $\mathrm{H}$, et al. High density lipoprotein structural changes and drug response in lipidomic profiles following the long-term fenofibrate therapy in the FIELD substudy. PLoS ONE 2011;6:e23589.

38. Kaddurah-Daouk R, Baillie RA, Zhu H, Zeng Z-B, Wiest MM, Nguyen UT, et al. Lipidomic analysis of variation in response to simvastatin in the Cholesterol and Pharmacogenetics Study. Metabolomics 2010;6:191-201.

39. Azmi AS, Bao B, Sarkar FH. Exosomes in cancer development, metastasis, and drug resistance: a comprehensive review. Cancer Metastasis Rev 2013;32:623-42.

40. Vlassov AV, Magdaleno S, Setterquist R, Conrad R. Exosomes: current knowledge of their composition, biological functions, and diagnostic and therapeutic potentials. Biochim Biophys Acta 2012;1820:940-8.

41. Record M, Poirot M, Silvente-Poirot S. Emerging concepts on the role of exosomes in lipid metabolic diseases. Biochimie 2014;96:67-74.

42. Record M, Carayon K, Poirot M, Silvente-Poirot S. Exosomes as new vesicular lipid transporters involved in cell-cell communication and various pathophysiologies. Biochim Biophys Acta 2014;1841:108-20. 